\title{
OBLIQUE SHOCKS AS THE ORIGIN OF RADIO TO GAMMA-RAY VARIABILITY IN ACTIVE GALACTIC NUCLEI
}

\author{
Philip A. Hughes, Margo F. Aller, and Hugh D. Aller \\ Astronomy Department, University of Michigan, Ann Arbor, MI 48109-1042, USA; phughes@umich.edu, mfa@umich.edu, haller@umich.edu \\ Received 2010 December 14; accepted 2011 April 20; published 2011 June 20
}

\begin{abstract}
The "shock in jet" model for centimeter-waveband blazar variability is revisited, allowing for arbitrary shock orientation with respect to the jet flow direction, and both random and ordered magnetic field. It is shown that oblique shocks can explain events with swings in polarization position angle much less than the $90^{\circ}$ associated with transverse structures, while retaining the general characteristics of outbursts, including spectral behavior and level of peak percentage polarization. Models dominated by a force-free, minimum energy magnetic field configuration (essentially helical) display a shallow rise in percentage polarization and frequency-dependent swing in polarization position angle not in agreement with the results of single-dish monitoring observations, implying that the field is predominantly random in the quiescent state. Outbursts well explained by the "shock in jet" model are present during $\gamma$-ray flaring in several sources, supporting the idea that shock events are responsible for activity from the radio to $\gamma$-ray bands.
\end{abstract}

Key words: galaxies: jets - magnetic fields - polarization - radiation mechanisms: non-thermal - shock waves

\section{INTRODUCTION}

One of the early results from EGRET was the discovery that a subset of very active extragalactic objects, blazars, emits at $\gamma$-ray energies. A close association between activity in the radio band identified using total flux density monitoring observations and detections by EGRET in the GeV band was established in the mid-1990s, e.g., Valtaoja \& Teräsranta (1995), an association which has been confirmed using the large data base of measurements provided by Fermi and monitoring measurements from both single dish and Very Long Baseline Array (VLBA) imaging measurements, e.g., Kovalev et al. (2009); Richards et al. (2010). For the past 25 years, the accepted explanation for blazar variability in the optical-to-radio bands has been the "shock in jet" model; hence, the temporal associations between the activity in the radio and $\gamma$-ray bands and the fact that the EGRET detections occurred during the rise portions of the radio flares suggested to investigators that the same shocks producing the radio flares were responsible for the emission in the $\gamma$-ray band, e.g., Valtaoja \& Teräsranta (1996). This shock scenario has been echoed in recent studies of Fermi observations of active galactic nuclei (AGNs), e.g., Abdo et al. (2010b), but rigorous tests have not been extensively carried out.

Evidence in support of the "shock in jet" scenario originally came from model fits to the broadband spectral evolution in the optical-to-radio band in 3C 273 (Marscher \& Gear 1985) and independently from radiative transfer model fits to multifrequency total flux density and linear polarization monitoring measurements in the centimeter band (Hughes et al. 1989a, 1989b, 1991). The generally accepted scenario is that outbursts result from instabilities which develop naturally within jet flows, producing shocks. The magnetic field is initially random within this emitting region (Jones et al. 1985), but the shocks produce a compression and an increased ordered component; the expected signature in the radio band linear polarization light curves for such a shock event is a swing in the electric vector position angle (EVPA, an orientation orthogonal to the magnetic field direction in a transparent source) and an increase in the fractional linear polarization.
This early modeling assumed that the shocks had a specific orientation-transverse to the flow direction. Attempts to fit later radio band events in these same sources with the same model parameters used successfully in the original fits, however, failed, and the characteristic behavior of the variations suggested that shocks are more generally oriented obliquely to the jet flow direction; thus, the initial transverse shocks identified from their well-resolved, distinct appearance represented a special case of a more general phenomenon. Conical shocks have been discussed by Cawthorne \& Cobb (1990), and the formulation presented therein has been applied to the data for some sources, e.g., Marscher \& Jorstad (2011).

Support for the shock model of major outbursts seen in singledish data, and the propagating components seen in maps of parsec-scale flows, plus support for a shock explanation for at least some Fermi events, would come from (1) "revalidating" the "shock in jet" model, by showing that oblique shocks can indeed explain the commonly observed reduced swing in EVPA through only tens of degrees, and associated increases in both fractional polarization and total flux density (flares) with the spectral behavior exhibited in the data; (2) showing that $\gamma$-ray flares occur at the same time as radio band events plausibly explained by an oblique shock model. It is also desirable to test whether the single-dish data can discriminate between models involving purely random magnetic field, purely ordered magnetic field, or a mixture of the two. To those ends it is illustrated here that the salient features identified in radio band linear polarization and total flux density observations obtained by the University of Michigan monitoring program (hereafter UMRAO) are reproduced by simulations using plausible input parameters in modeling involving radiative transfer through flows with propagating, oblique shocks; and it is demonstrated that this shock signature is present during $\gamma$-ray flaring in several sources.

In Section 2 the results from the Michigan variability program are summarized, the general characteristics of the centimeterwaveband variability are defined, and their relation to $\gamma$-ray flaring is discussed, with examples. In Section 3 the details of an oblique shock model for this centimeter-waveband activity are set out: the quiescent flow, models for both random and ordered 
magnetic field components, the structure of an oblique shock, and its propagation. The essential elements of an existing code for the transfer of polarized radiation are recapped in Section 4, and the results of applying this to a flow with representative flow parameters are presented in Section 5: results for a range of observer orientations and magnetic field topologies are contrasted. Section 6 summarizes and discusses the findings that oblique shocks can explain the observed centimeter-waveband events, the behavior of which support a model with at most a modest contribution from an ordered magnetic field component.

\section{OBSERVATIONS}

\subsection{The UMRAO Database}

As part of the Michigan variability program carried out with the $26 \mathrm{~m}$ radio telescope, source-integrated, single-dish total flux density, and linear polarization measurements have been obtained for hundreds of sources, extending over time windows of up to four decades. The data acquired provide a comprehensive view of the properties of blazar variability in the centimeter band based on long-term data acquired and reduced in a consistent and uniform manner. These measurements commenced at $8 \mathrm{GHz}$ in the mid-1960s, at $14.5 \mathrm{GHz}$ in 1974 and at $4.8 \mathrm{GHz}$ in 1978 . The number of sources included in the UMRAO monitoring program increased dramatically in the late 1970s when an automated observing program was implemented; subsequently the fraction of time utilized per year for AGN observations increased to over $90 \%$. Typically 20-25 sources are observed in a $24 \mathrm{hr}$ period; each observation consists of a series of on-off measurements over approximately 30-45 minutes. Measurements of calibrators are interspersed with observations of program sources every $11 / 2$ to $2 \mathrm{hr}$. In general, the observing cadence adopted for each program source is adjusted to follow the variability in that particular AGN. Very active sources such as those included in the shock program described here are typically observed 1-2 times per week at $14.5 \mathrm{GHz}$ and once per week at 8 and $4.8 \mathrm{GHz}$ during flaring periods. This cadence is required to follow the rapid variations in linear polarization expected from past observations.

\subsection{Characteristics of the Variability}

Studies of the data for both individual sources and for statistical samples have identified a number of general properties of the variability: the characteristic variability timescales of the total flux density, identified from first-order structure analyses, are of the order of two years in the observer's frame with some spread, e.g., Hughes et al. (1992). The ratios of peak to quiescent amplitude of these variations are characteristically only a factor of a few, reaching at most a factor of eight. While numerous attempts have been made to identify periodic behavior in the total flux density light curves, in general consecutive events do not repeat. The outburst shapes are characterized by a rapid rise followed by a more gradual decline. In the millimeter band, the Metsähovi monitoring data have been successfully fit assuming a function with an exponential rise, a sharp turnover, and an exponential decay with a timescale approximately 1.3 times longer than the rise timescale (Valtaoja et al. 1999), but a generic shape is not apparent in the events observed in the lower frequency UMRAO data, possibly due to the blending of individual events. The total flux density spectra in the centimeter band are typically inverted and relatively flat $(|\alpha| \leqslant 0.5)$; however, these differ from source to source and can differ from event to event in the same source. The spectrum

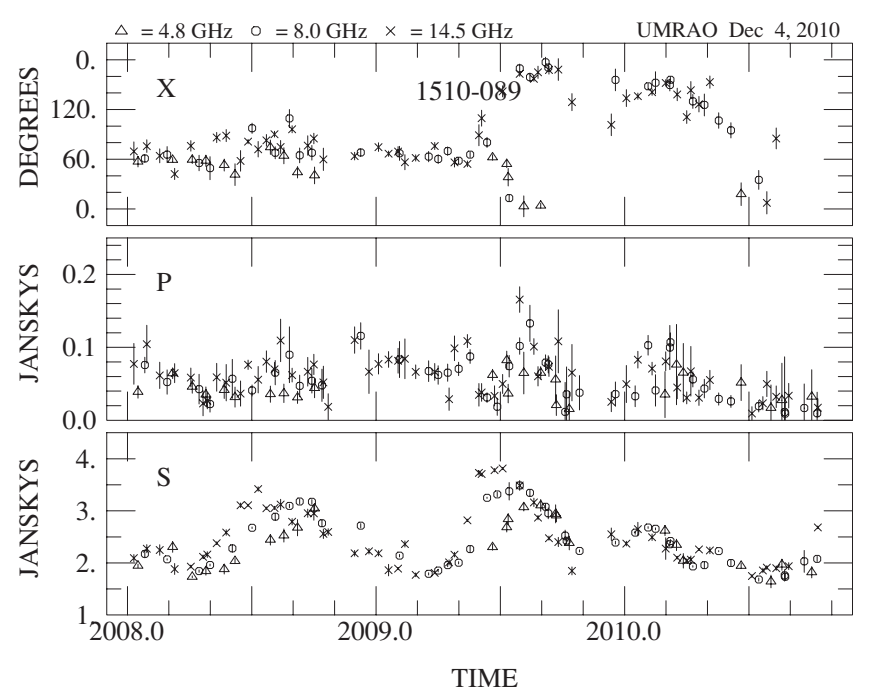

Figure 1. From bottom to top: daily averages of the total flux density, the linearly polarized flux density, and the electric vector position angle in PKS 1510-089. The data at $14.5,8.0$, and $4.8 \mathrm{GHz}$ are denoted by crosses, circles, and triangles, respectively. The radio band flaring shown is temporally associated with $\gamma$-ray activity. Note the differences in spectral behavior during the flaring periods shown.

itself in many cases evolves during an outburst; in many large events the spectrum is inverted until burst maximum and flattens during outburst decline. Associated flares are often apparent in polarized flux density, and in some cases individual events can be seen in linear polarization which appear as blended events in total flux density.

Generic properties of the variability in total flux density and linear polarization are summarized in Table 1(A), for comparison with models to be presented in Section 5. The values listed are based on examination of the UMRAO database, while Figures 1-3 present specific examples illustrating the range in these properties during recent well-resolved events temporally associated with $\gamma$-ray flares detected by Fermi. The table contains: in Column 2, a simple measure of the flare shape given by the rise time, i.e., the time from start to peak, $\tau_{\text {rise }}$, divided by the event duration, $T$; in Column 3 the spectral index at outburst start, at $14.5 \mathrm{GHz}$ peak, and at end, in the UMRAO frequency range 4.8-14.5 GHz; in Column 4 , characteristic values of the maximum fractional linear polarization (note that while the values listed are typical, values near $18 \%$ have occasionally been observed during flares); in Column 5 the monotonic swing in EVPA during an event, $\triangle \mathrm{EVPA}_{\text {time }}$; and in Column 6 , the spectral variation in EVPA throughout this swing, $\triangle \mathrm{EVPA}_{\text {freq }}$. The spectral indices follow the sign convention $S_{v} \propto v^{+\alpha}$, and the values listed for this parameter are based on numerical results for selected events, using paired values of two-week averages of the data at 14.5 and $4.8 \mathrm{GHz}$. For linear polarization (peak fractional linear polarization and variation in EVPA with time or frequency), the values listed were determined from visual inspection of the long-term UMRAO data. No corrections were applied for Faraday rotation; but events in sources with low source-integrated Faraday rotation measure were preferentially examined.

Figure 1 shows the UMRAO monitoring data for the QSO PKS 1510-089. A series of $\gamma$-ray flares occurred during the time period shown which have been analyzed in detail in Abdo et al. (2010a) and in Marscher et al. (2010). A particularly large and isolated event was identified with start date 2009 March 10 and stop date 2009 April 9 (Abdo et al. 2010a). The UMRAO 
Table 1

Typical Flare Properties

\begin{tabular}{lccccc}
\hline \hline Frequency/Time & $\tau_{\text {rise }} / T$ & $\alpha$ & $P \%_{\max }$ & $\Delta \mathrm{EVPA}_{\text {time }}$ & $\Delta \mathrm{EVPA}_{\text {freq }}$ \\
\hline $14.5 \mathrm{GHz}$ & & A. UMRAO Data & \\
8.0 & 0.5 & 7 & \\
4.8 & 0.6 & 4 & \\
& 0.7 & 3.5 &
\end{tabular}

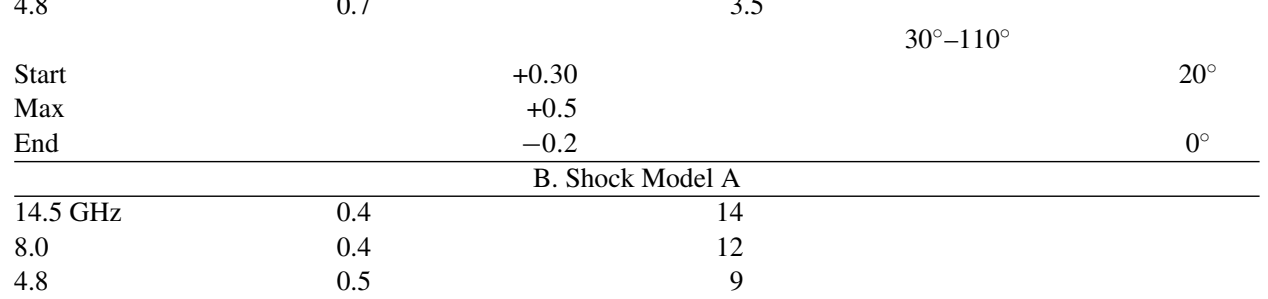

\begin{tabular}{|c|c|c|c|c|c|}
\hline 4.8 & 0.5 & & 9 & $25^{\circ}$ & \\
\hline Start & & -0.11 & & & $4^{\circ}$ \\
\hline Max & & +0.13 & & & 1.5 \\
\hline End & & -0.13 & & & $4^{\circ}$ \\
\hline \multicolumn{6}{|c|}{ C. Shock Model C1 $\left(\eta=90^{\circ}, \theta_{\mathrm{obs}}=20^{\circ}\right)$} \\
\hline$\overline{14.5 \mathrm{GHz}}$ & 0.4 & & 14 & & \\
\hline 8.0 & 0.4 & & 12.5 & & \\
\hline \multirow[t]{2}{*}{4.8} & 0.4 & & 9.5 & & \\
\hline & & & & $85^{\circ}$ & \\
\hline Start & & -0.10 & & & $7^{\circ}$ \\
\hline Max & & +0.07 & & & 1.5 \\
\hline End & & -0.11 & & & $19^{\circ}$ \\
\hline
\end{tabular}

Note. Specific values of $\alpha$ vary from event to event. Those listed show a typical change during an observed flare.

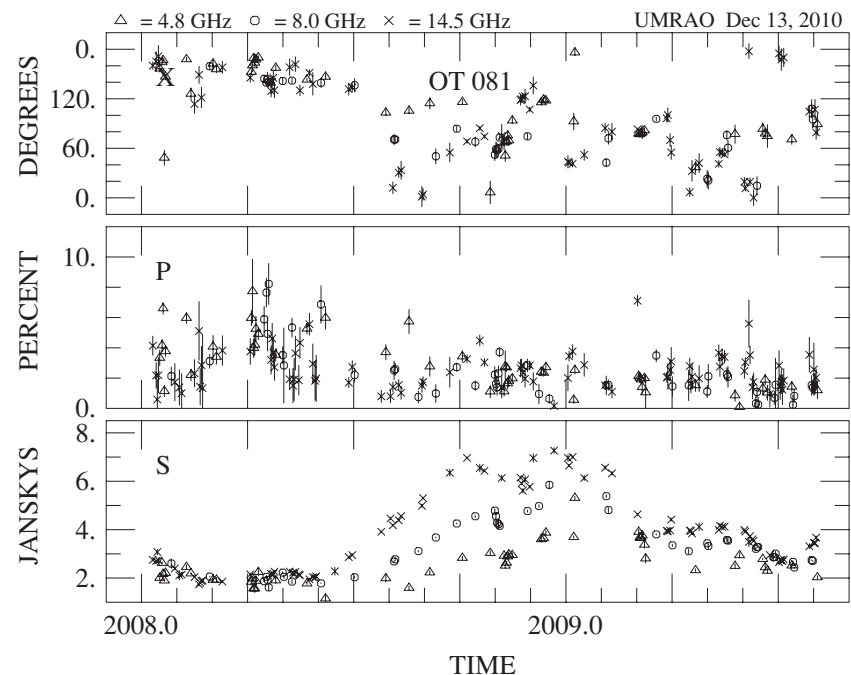

Figure 2. From bottom to top: daily averages of the total flux density, the fractional linear polarization, and the electric vector position angle for OT 081 (1749+096). Symbols are as in Figure 1.

light curves reveal that a radio band total flux density flare commenced in early 2009 at which time both a resolved linear polarization flare and an ordered swing in EVPA are apparent at 14.5 and $8.0 \mathrm{GHz}$. Because of the differences in variability timescales in the radio and $\gamma$-ray bands and the expected time delays produced by self-absorption in the emitting region, there are some uncertainties in associating specific events across bands; an event with the signature of an oblique shock, however, unambiguously occurred during this time period which includes the $\gamma$-ray flare.

Figure 2 shows the radio band data during the period 2008.0 through 2009.5 for the very active BL Lac object, OT 081 $(1749+096)$, one of the three sources originally modeled using the transverse shock formulation (Hughes et al. 1991). Several resolved events are apparent in both the total flux density and fractional linear polarization light curves; an ordered swing in EVPA at $14.5 \mathrm{GHz}$ occurred from 2008 August to December followed by a shorter-duration ordered swing during 2009 January-February. The Fermi light curve for this source is included in the variability study based on data from the first year of Fermi operation (Abdo et al. 2010b). A very large flare in the $\gamma$-ray band was in progress in 2008 August, and a second flare which peaked in 2009 mid-March is also apparent in these data. Activity, including strong flaring, occurred in both the $\gamma$-ray and radio wavebands.

Figure 3 shows both the $\gamma$-ray and radio band light curves for OJ 287. In this source the signature of a shock temporally associated with the outburst observed by Fermi is clearly apparent. The swing in EVPA through a limited range of about $40^{\circ}$ is consistent with the passage of an oblique shock.

\section{JET MODEL}

\subsection{The Quiescent Flow}

All computations are performed in a box $61 \times 61 \times 600$ cells in extent, with the axis of the jet parallel to the long axis, $z$, and polar angles are measured in the usual sense: $\theta$ from the $z$-direction and $\phi$ from the $x$-direction. A simple conical form has been taken for the jet boundary as observations cannot constrain more complex profiles explored over at most tens of jet radii. The opening angle $(\mu)$ is determined by setting the jet radius to be $r_{\text {lo }}$ on the inflow plane $z=0$ and $r_{\text {hi }}$ on the outflow plane $z=z_{\max }=600$; angles are typically $1^{\circ}-2^{\circ}$.

Quiescent flow values are established across the entire domain, and then an apodizing filter,

$$
a(\rho)=0.5\left[1+\tanh \left(\frac{-\rho+r(z)}{a_{w}}\right)\right],
$$




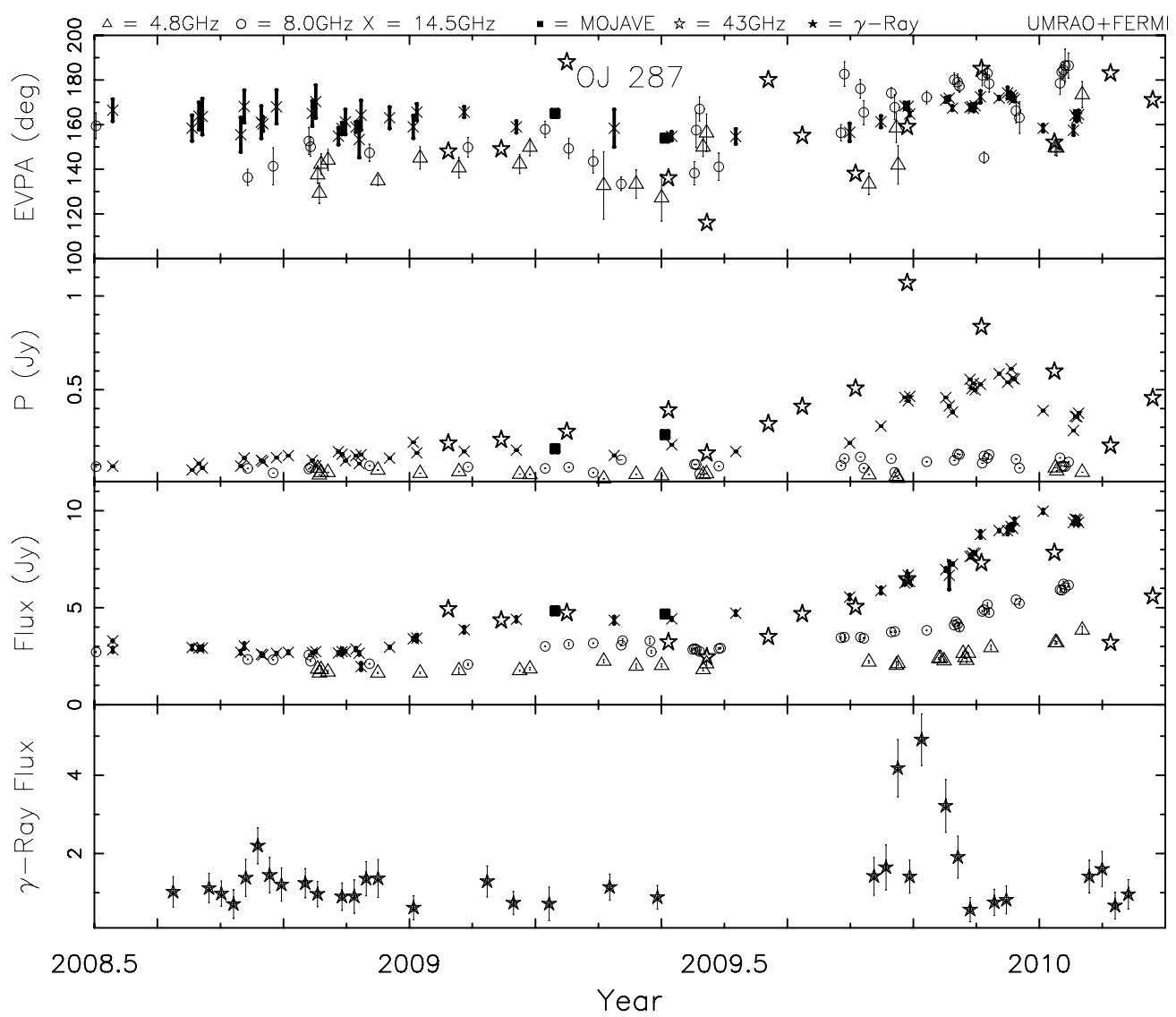

Figure 3. From bottom to top: $\gamma$-ray light curve and daily averages of the total flux density, the linearly polarized flux density, and the electric vector position angle for OJ $287(0851+202)$. Symbols are as in Figure 1 for the UMRAO monitoring data; source-integrated VLBA data obtained from the MOJAVE (15 GHz) and BU $(43 \mathrm{GHz})$ Web sites are shown for comparison. The $\gamma$-ray light curve was kindly provided by S. Jorstad. Units are photons s $\mathrm{cm}^{-2} \times 10^{-7}$.

is applied, where $\rho=\left(x^{2}+y^{2}\right)^{1 / 2}$ is the radial location of a cell at some $z$, where the jet radius is $r(z)$, and $a_{w}$ is a parameter that controls the flow's boundary extent; $a_{w}$ is typically one cell.

Whether flows exhibit significant acceleration or deceleration remains controversial, and indeed, might differ from source to source. Deceleration has been inferred for FR 1 radio galaxies (Laing 1996; Laing et al. 1999), while a recent study of the BL Lac object OJ 287 finds that the flow remains highly relativistic to distances as high as hundreds of kiloparsecs from the nucleus (Marscher \& Jorstad 2011). In view of this uncertainty, and the limited number of jet radii explored, the quiescent flow speed is taken as a constant specified by its Lorentz factor, $\gamma_{f}$; values are typically $2-5$. A diverging flow is then established, with stream lines parallel to the $z$-axis on axis, and parallel to the flow boundary there. The magnetic field is constructed as described in Section 3.2 and assumed to fall in strength along the flow as $r(z)^{-2}$. Leahy (1991) gives a good overview of the evolution of parameters in a diverging adiabatic flow, and it might be thought that a fall-off as $r(z)^{-4 / 3}$ would be appropriate for a flow of fixed speed, given that the default assumption is that of a random field. However, that assumes no coupling between the components, so that the component perpendicular to the flow falls more slowly, driving the flow away from isotropic turbulence. The assumption of an isotropic random component at every location in the quiescent flow implies a turbulent driving that persists the length of the flow, transforming perpendicular into parallel field, which declines with the assumed $r(z)^{-2}$ dependence, and vice versa, coupling the decline of the perpendicular component to that of the parallel component. Further, the helical ordered field that is also explored, if generated from a turbulent component by a dissipative dynamo process such as noted in Section 3.2.2, will then follow the same global trend. The adopted dependence is thus a simplification, but reasonable given the other approximations. As discussed in the next section, the method used to generate a random field component, the ad hoc scaling along the flow, and the scaling of the relative strengths of random and ordered field components mean that the field does not satisfy a self-consistent magnetohydrodynamic model for its generation and is not divergence-free. However, the aim is to construct a plausible magnetic field topology with which to explore the properties of the emergent radiation, not a field to be evolved subject to constraints to ensure conservation and/or preservation of the divergence-free character of the field.

The flow is assumed to be pervaded by a power-law distribution of radiating particles, with density

$$
n(\gamma) d \gamma=n_{0} \gamma^{-\delta} d \gamma, \quad \gamma>\gamma_{i},
$$

with the index $\delta$ fixed. Adiabatic gains and losses will not change the slope, synchrotron losses are ignored over the tens of jet radii propagation explored here, and no allowance is made for Fermi acceleration at shocks, whose primary role will be to produce a small number of particles radiating at frequencies above those probing the quiescent emission. The absolute scaling of the density is arbitrary, as functions of the density constant, $n_{0}$, and the magnetic field strength are absorbed into a fiducial optical depth, $\tau$, which acts as a free parameter; see Section 4 . The density constant, $n_{0}$, is taken to fall due to adiabatic expansion of the flow according to $r(z)^{-2(\delta+2) / 3}$, 
and the cutoff thermal Lorentz factor (see Section 4 for the significance of this quantity), $\gamma_{i}$, falls as $r(z)^{-2 / 3}$.

\subsection{Magnetic Field Structure}

Arguments in favor of a largely tangled magnetic field were made by Hughes (2005), the salient points being: (1) the low degree of polarization exhibited by compact extragalactic radio sources when in a quiescent state has been widely interpreted as due to "root-N" depolarization in a synchrotron source with many randomly oriented magnetic cells; (2) there have been successful models of the temporal, spatial, and spectral attributes of outbursts in a number of individual sources, with a scenario in which the shock compression of a flow provides an effective order to the otherwise random magnetic field; (3) the observed levels of linear and circular polarization are best modeled by a scenario in which root- $\mathrm{N}$ depolarization plays a significant role, albeit that some of the magnetic energy is in an ordered component.

In contrast to this picture, Gabuzda and coworkers (Gabuzda et al. 2004, 2005; Mahmud \& Gabuzda 2008; O'Sullivan \& Gabuzda 2008; Contopoulos et al. 2009) have argued in favor of a large-scale magnetic field-in particular with a helical character-the key evidence being rotation-measure gradients across jets, which are interpreted as due to the systematic change in the line-of-sight component of the jet magnetic field. Furthermore, it is almost invariably the case that "central engine" models invoke an ordered magnetic field in the environment of a supermassive black hole (see, by way of example, Komissarov et al. 2007; McKinney \& Narayan 2007) and this order (together with that due to shear) would be expected to be imprinted upon the parsec-scale flow. Recent work by Broderick \& McKinney (2010) and Taylor \& Zavala (2010) notes that the presence of a helical field does not necessarily imply that a simple monotonic variation in rotation measure across the flow will be observed, and the resolution currently available precludes definitively establishing the character of any ordered field within the jet. However, clearly there is evidence consistent with such a field in a number of sources.

If an ordered field exists, and its sense is determined by the spin of the central black hole, and/or the rotation of the associated accretion disk, then the sign of the resultant circular polarization would be expected to be a fixed attribute of any source. However, Stokes $V$ monitoring does not provide a simple picture; while, some sources exhibit such a constancy, in others changes in the handedness of circular polarization with time and frequency are quite unambiguous over durations of months to years, e.g., Aller et al. (2010). Also, evidence for a reversal in the direction of the rotation-measure gradient across the jet of $1803+784$ presented by Mahmud et al. (2009), at large distance from the core and away from the portion of the jet most affected by finite beam size, are at odds with the results of simulations by Broderick \& McKinney (2010), who argue against the "magnetic tower" model for a temporal change in the sign of the rotation measure. The analysis of all-Stokes polarization data for 3C 279 (Homan et al. 2009) also does not find support for the role of a helical field in the production of circularly polarized emission. Some resolution of these conflicting perspectives may yet come from a more careful consideration of the emission location (the jet spine) and the source of Faraday rotation (the jet sheath; the dominant contribution to Faraday effects in the models of Broderick \& McKinney 2010). Until higher spatial resolution data become available, and simulations can track the distribution of radiating particles, the topology of the magnetic field remains uncertain, and a contentious issue, and so here both random and ordered fields are considered.

\subsubsection{Random Field Component}

An elegant method of establishing a random magnetic field is to select random phases, and random amplitudes from a Rayleigh distribution, for the Fourier transform of the vector potential, $\tilde{\mathbf{A}}(\mathbf{k})$. The Fourier transform of the magnetic field is then $\tilde{\mathbf{B}}(\mathbf{k})=i \mathbf{k} \times \tilde{\mathbf{A}}(\mathbf{k})$, and an inverse Fourier transform of this yields a magnetic field that is guaranteed to satisfy the divergence-free constraint. (See, e.g., Tribble 1991 for a detailed exposition of this approach.) However, as first noted in this context by Jones (1988), such a technique leads to large-scale Fourier components that give rise to a small but significant excess polarized flux density. An exploration of this in the current study showed that unacceptable levels of polarized flux density were produced, and that there is no simple way to mitigate that effect.

Jones (1988) used a lattice of nested cells, each with randomly chosen field orientation, and with the amplitude of the field components scaled to produce an approximately Kolmogorov power spectrum. The goal of the current study is such that there is a need to extensively explore a very large parameter space, and thus use a three-dimensional grid of limited resolution, in order to keep individual runs to a modest time. Furthermore, a jet length of many jet radii is required, because although the total flux density falls rapidly after a propagating enhancement to the particle and field densities passes the $\tau=1$ surface, a polarized flux density significantly above the quiescent value persists for much longer. Thus there are too few cells within the jet to effectively employ a multi-scale approach, and simply assign randomly chosen field directions within each cell. In principle the field should be advected with the flow, but such advection cannot be handled self-consistently at oblique shocks, due to the simple way that those structures are modeled (see Section 3.4), and as this study is concerned with "macroscopic" behavior in the polarized emission, not random fluctuations arising from flow structures lighting up different realizations of random field, a static random field is built into the jet.

To reduce the computational overhead, multiple realizations of a random field have not been generated for each parameter combination. However, in Section 5.2 radiation transfer is performed for a range of azimuthal angles about a flow with propagating oblique shock. By symmetry the results for azimuthal angles $225^{\circ}, 270^{\circ}$, and $315^{\circ}$ are identical to results for $135^{\circ}, 90^{\circ}$, and $45^{\circ}$, respectively, except that the different angles sample different projections of the random field component, and so the differences in emergent radiation for the three pairs of runs provide insight into variations to be expected from different realizations. At all azimuthal angles, total intensity curves are identical, as are those for percentage polarization, but during outburst there are small differences in EVPA as a function of frequency; this is greatest at the first time shown, the quiescent state, because of the low level of polarized emission then.

\subsubsection{Ordered Field Component}

As discussed above, ordered, and in particular "helical," magnetic fields have received much attention over the last decade. In order to include the most physically plausible ordered field component, with the least number of free parameters, a force-free, minimum energy configuration is adopted. On the 
sub-parsec scale of interest here, the flow is far enough from the central engine that local evolutionary effects should dominate over the influence of the engine's ergosphere or inner accretion disk.

Indeed, long-term changes in the character of a large-scale magnetic field are possible, if jets are subjected to turbulence and a dissipative dynamo process. Turbulence could be driven throughout the body of the flow due to their ultra-high Reynolds number. Mean field dynamos in sheared, turbulent flows are known to occur (Rogachevskii \& Kleeorin 2003), and although the process has not been explored in cylindrical geometry, it can be speculated that the mean field then takes a form similar to that of a force-free flux tube. This field exists in what would be the asymptotic region discussed by McKinney (2006), and its symmetry is thus unrelated to the angular momentum of the "central engine." Although the process of field reversal is different from that exhibited by the solar-terrestrial field-being stochastic rather than periodic-recent simulations (Yousef et al. 2008) have shown such behavior, and it would naturally explain the observed circular polarization "flips."

Whether the field evolves due to dynamo action or relaxation, a likely end point is a force-free configuration $\nabla \times \mathbf{B}=\sigma \mathbf{B}$ (Eilek \& Hughes 1991). It has been shown by Königl \& Choudhuri (1985) that in cylindrical geometry, only the modes $m=0$ and $m=1$ contribute to the general solution to the force-free equation in the minimum energy state. For a range of jet and ambient medium parameters, and for any parameters leading to a jet with small deviation from axisymmetry, only the $m=0$ mode need be considered. Then, by the divergence-free constraint $\nabla \cdot \mathbf{B}=0$, the axial wavenumber $k=0$.

In circular cylindrical coordinates, $(\rho, \phi, z)$, this leads to a field configuration

$$
\mathbf{B}=B_{0}\left(0, J_{1}(K \rho), J_{0}(K \rho)\right),
$$

where the $J$ are Bessel functions of integer order and $K$ is a constant that must be determined. The only physical boundary condition is that the radial component of $\mathbf{B}$ vanishes there, but as this is identically zero for the $m=0$ mode, this boundary condition provides no constraint. By choosing $K=2.41 / r(z)$, with $r(z)$ the jet radius, the axial field goes through a null at the boundary (Lundquist 1950). While it is in principle permissible for the axial field to reverse sign inside the jet, the adopted value prevents that, and is consistent with the simple field topology selected by evolution to a force-free, minimum energy state. This field is adopted "locally" in that the jet radius increases due to flow divergence, but note that it is not built self-consistently into the prescription of the magnetic field.

The constant $B_{0}$ is varied to model flow divergence and shock compression. The strength of the random component is established by scaling so that $\left\langle\mathbf{B}_{\mathrm{ran}}^{2}\right\rangle$ is a specified multiple of the ordered field strength. In the absence of a fully selfconsistent magnetohydrodynamic model, it is not clear whether the random field strength should be a multiple of the local ordered field strength (corresponding, for example, to a turbulent generation of the former from the latter by a fixed number of eddy turnovers); or spatially fixed for a given jet radius, a multiple of the axial ordered field strength (corresponding, for example, to a turbulent generation of the former to fixed multiple of the kinetic energy density in flow turbulence). Both approaches have been tried, revealing that the choice has no impact on the final results, merely changing the precise value of $f^{2}=\mathbf{B}_{\text {ord }}^{2} /\left\langle\mathbf{B}_{\text {ran }}^{2}\right\rangle \sim 1$ where a transition in the characteristic behavior of the polarized flux density is evident.

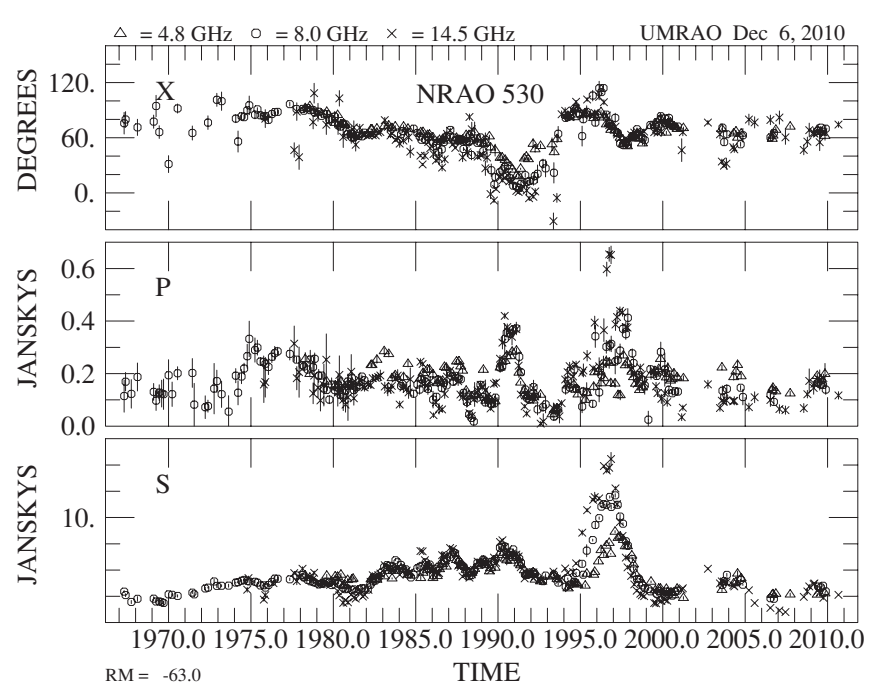

Figure 4. From bottom to top: 60 day averages of the total flux density, the linear polarization, and the electric vector position angle for NRAO 530. Symbols are as in Figure 1. A rotation measure of $-63 \mathrm{rad} \mathrm{m}^{-2}$ has been assumed (Rusk 1988). The Faraday-corrected EVPA is near $70^{\circ}$ at $14.5 \mathrm{GHz}$ during the relatively quiescent period since circa 2000 following a very large outburst in the 1990s. The position angle of the jet determined from MOJAVE measurements at the same frequency is $12^{\circ}$ (Kovalev et al. 2005).

\subsection{Axial Magnetic Field}

It is extraordinarily difficult to define a quiescent state from data. The UMRAO monitoring data exhibit almost continuous activity, and even apparently inactive phases may be periods in which small-amplitude outbursts are temporally unresolved. VLBI monitoring similarly reveals few if any inactive epochs, and apparently quiescent flows may contain spatially unresolved components. The UMRAO database has been searched to identify periods of "quiescence," during which there is measurable linearly polarized emission. Between 1998 and $20010735+178$ exhibited a low level of constant flux density at the UMRAO frequencies, with $\sim 2 \%$ polarization and EVPA implying an ordered magnetic field lying within some tens of degrees of the jet direction determined from MOJAVE data (Lister et al. 2009). Similar numbers apply to NRAO 530 from 2002 to 2010 as illustrated in Figure 4. Based on (admittedly sparse) examples such as these, it is plausible to assume that in the quiescent state a weak axial field is added to any random component, and in all simulations that follow, an axial mean field with $2 \%$ the energy density of the random component is added. This establishes a well-defined EVPA in the quiescent state.

\subsection{Oblique Shocks}

Modeling a transverse structure comprising forward and reverse shocks, separated by a contact discontinuity, is straightforward, as no lateral flow is required: the shocked flow domain expands in the frame of the contact surface, and either the expansion may be ignored during the brief interval that the structure traverses the $\tau=1$ surface, or the expansion can be modeled self-consistently from the jump conditions. Furthermore, either the forward or reverse shocked flow can be assumed to dominate emission, or emission from both regions can be included.

A kinematic model for an oblique shock is difficult to construct, because by its very nature, an oblique shock deflects the flow. Indeed, they have long been invoked to model flow curvature in radio jets (Smith 1984). Deflection of the flow would appear to be inconsistent with the simple, static, conical flow 
boundary assumed here. However, consider BL Lac as an example source: Denn et al. (2000) present extensive monitoring by VLBI which reveals knots with linear polarization behavior strongly suggestive of oblique shocks. While these authors stress the curvature of the knot trajectories, and difference between the kinematics of the four components studied, an overlay of the trajectories (as defined by the data, rather than the helical flow model) for components S7 and S9 exposes a remarkable similarity, while the trajectories of S9 and S10 differ significantly only at the last epoch of the later, and in that S10 may be followed closer to the core. While component S8 does follow a distinctly different, and more curved, trajectory, the general impression is of a well-defined channel with modest curvature amplified in projection that can support the propagation of multiple oblique structures. Furthermore, studies such as that by Hughes (2005) show that oblique shocks (which may be transient features) can form and propagate, filling the cross section of a flow, while that flow maintains a simple jet-like, rectilinear form.

An oblique shock is thus taken to be a plane spanning the jet, of obliquity $\eta$, measured with respect to the direction of the upstream flow, which is approximated as being in the sense of the jet axis, ignoring the small divergence of the stream lines. $\eta=90^{\circ}$ corresponds to a transverse shock, and the orientation of the shock is specified by the azimuthal direction of the shock normal, $\psi$. The shocked flow is a volume extending from this plane to a parallel plane, a distance $w$ along the flow axis. The limit of the shocked flow is fixed; it is on the "core" side of the shock plane for a "forward" shock (moving faster than the underlying jet flow), and on the other side for a "reverse" shock (being overtaken by the jet flow). The shocked flow may be terminated by adiabatic expansion of the shocked material, radiative losses within the flow, or simply reflect the distance the downstream flow has extended since formation. Adiabatic expansion is not relevant in this context in the current model, as by construction, the flow is constrained to lie within the quiescent jet boundary. Radiation losses could have imposed a frequency-dependent length on the downstream flow (Marscher \& Gear 1985) prior to evolution through the modeled domain, but as explained in Section 4, the UMRAO observing frequencies are close (being separated by $\sim \sqrt{3}$ ), and the length determined by radiation losses goes as $v^{-1 / 2}$, so little frequency dependence of the structure is expected. For the sake of definitiveness, it is assumed that the length of shocked flow is simply the evolved extent of the downstream flow, which changes little during the brief propagation through the modeled domain.

The shocked flow is characterized by a compression $\kappa<1$, and following Cawthorne \& Cobb (1990), the upstream flow speed in the rest frame of the shock, $\beta_{u}^{\prime}$ is computed:

$$
\begin{aligned}
\beta_{u}^{\prime 2}= & \left\{\kappa^{2} \sin ^{2} \eta-\cos ^{2} \eta-1+\left[\left(\kappa^{2} \sin ^{2} \eta-\cos ^{2} \eta-1\right)^{2}\right.\right. \\
& \left.\left.+4 \kappa^{2}\left(8 \sin ^{2} \eta+1\right) \sin ^{2} \eta-4 \cos ^{2} \eta\right]^{\frac{1}{2}}\right\} / \\
& \left\{2\left[\kappa^{2}\left(8 \sin ^{2} \eta+1\right) \sin ^{2} \eta-\cos ^{2} \eta\right]\right\}
\end{aligned}
$$

From Lind \& Blandford (1985) it follows that the downstream flow speed (also in the shock frame) is given by

$$
\beta_{d}^{\prime}=\frac{\left[\left(1-\beta_{u}^{\prime 2} \cos ^{2} \eta\right)^{2}+9 \beta_{u}^{\prime 4} \cos ^{2} \eta \sin ^{2} \eta\right]^{\frac{1}{2}}}{3 \beta_{u}^{\prime} \sin \eta}
$$

and the flow is deflected by angle $\zeta$ given by

$$
\tan \zeta=\frac{\tan ^{2} \eta\left(3 \beta_{u}^{\prime 2}-1\right)-\left(1-\beta_{u}^{\prime 2}\right)}{\tan \eta\left(\tan ^{2} \eta+1+2 \beta_{u}^{\prime 2}\right)} .
$$

The flow direction within the shocked region is modified by $\zeta$, the shock speed, $\beta_{s}$, is computed from the assumed quiescent flow speed $\left(\beta_{f}\right)$ and the upstream speed in the shock frame $\left(\beta^{\prime}{ }_{u}\right)$, and the downstream flow speed $\beta_{d}^{\prime}$ is transformed into the observer's frame, $\beta_{d}$. Within the shocked region of the jet, the particle density is increased by $\kappa^{-(\delta+2) / 3}$, and the magnetic field components projected on the shock plane are increased by the compression factor.

\subsection{Time Evolution}

As noted in Section 3.1, evidence for the acceleration or deceleration of flows is ambiguous, and comes primarily from the observed deviations from simple rectilinear motion displayed by a number of superluminal components. It is difficult to distinguish between an acceleration or deceleration of the bulk flow, and that of shock waves on the flow, in addition to which acceleration or deceleration might be in direction only, due to a (modest) curvature of the jet (see, for example, Marscher 2006).

In the absence of compelling evidence for shock acceleration or deceleration, the propagation of the shock is modeled by a simple constant displacement of the shocked region discussed in Section 3.4; radiation transfer is performed for the quiescent flow at the first time step, and then over the remaining $N-1 \sim 10$ time steps the center of the shocked region traverses the flow so that the shocked domain lies just within the inflow jet volume at the second time step, and would have left the jet volume after the last time step. The corresponding physical time will be determined by the shock speed $\left(\beta_{s}\right)$, computed as described in Section 3.4, and the physical length of the flow; as the latter is subject to an arbitrary scaling, the time evolution is arbitrarily scaled to match typical outburst periods seen in the UMRAO data.

\section{RADIATION TRANSFER}

The transfer of polarized radiation is performed using the method described by Hughes (2005) and is briefly recapped here. It is based on a formulation for the transfer of polarized radiation through a diffuse plasma, allowing for emission, absorption, the birefringence effects of Faraday rotation and mode conversion (which can produce modest levels of circular polarization), and relativistic aberration and boost, which has been described in detail by a number of authors, and is compactly summarized by Jones (1988).

The observer lies at arbitrary polar angles $(\theta, \phi)$, defined in the conventional sense with respect to the Cartesian system used to describe the kinematic model. An array of lines of sight is established, using a preset density of lines along the longest axis of the projection of the computational volume on the plane of the sky, with a commensurate number orthogonal to that, to ensure equal resolution in the two directions. For the results presented below, a resolution of 128 pixels along the longest axis was used. For each line of sight, the algorithm finds the most distant cell, and radiative transfer is performed, cell to cell, until the "near side" of the volume is exited. Within each cell, an aberrated magnetic field direction is computed from the rest frame field, velocity, and observer location and is used with transfer coefficients modified by the relevant Doppler boost. 
For a given epoch of "observation" cell values corresponding to the appropriate retarded time should be used. However, as this leads to an enormous increase in the computational overhead, and can lead to simulated VLBI maps that are very difficult to interpret, because of the problem of unambiguously associating map features with physical flow structures, the radiation transfer is initially performed without retarded time effects included, and the latter will be considered only when critical to understanding particular features of light curves or maps. Simulations to justify this strategy are presented in Section 5.1.

Radiation transfer is performed at dimensionless observing frequencies of $0.6,1.0$, and 1.8125 corresponding to the UMRAO values of 4.8, 8.0, and $14.5 \mathrm{GHz}$. As described in Hughes et al. (1989a) a fiducial thermal ${ }^{1}$ Lorentz factor $\left(\gamma_{c}\right.$; "thermal" implies the Lorentz factor associated with the random motion of the emitting particles, as opposed to the bulk flow Lorentz factor) is adopted, which is the energy of those particles radiating at the central observing frequency in the average magnetic field for the jet volume at the first time step of evolution. The value of this fiducial Lorentz factor is not in itself significant, but model fitting to the data constrains how far below this the power-law electron energy distribution extends (to $\gamma_{i}$ ), because it is the presence of these lower energy, but still relativistic, particles that produce Faraday effects in the absence of a "cold" particle distribution; adopting a plausible fiducial value thus enables us to estimate the low energy cutoff to the power-law distribution.

Prior to the radiation transfer, the optical depth for each cell must be known; this is computed from the dimensionless line-ofsight length through the cell, cell magnetic field, and cell particle density. This is scaled with a single adjustable parameter chosen to produce some "target" optical depth through the entire volume at the central observing frequency, in the average magnetic field and particle density for the jet volume at the first time step of evolution. An initial "target" optical depth is chosen in light of the spectral characteristics of the data being modeled; the value is then adjusted to fine-tune the model fit. Given the actual observing frequencies, the choice of fiducial Lorentz factor implies a particular magnetic field strength; given that, and the optical depth needed to reproduce the data, knowledge of the physical scale of the jet implies a value for the particle density or vice versa. However, as the concern here is only with learning what such model fitting can say about the topology and orientation of observer, shock, and magnetic field, the implied field and particle densities are not explored.

\section{MODEL RESULTS}

\subsection{Role of Time Delay}

As discussed in Section 1, our primary goal is to "revalidate" the "shock in jet" model, demonstrating that oblique shocks retain the temporal and spectral characteristics of the total and polarized flux density behavior of centimeter-band outbursts previously modeled with transverse structures, while accommodating more complex behavior of the EVPA. As "proof of concept" parameters are chosen similar to those used to model outbursts in BL Lac (Hughes et al. 1989b), using a bulk flow of Lorentz factor $\gamma_{f}=2.5$ and compression $\kappa=0.7$, but with a shock oriented at $45^{\circ}$ to the flow axis, with the shock deflection in the plane of the sky; see Run A in Table 2.

\footnotetext{
This does not imply a thermal distribution of particle energies.
}

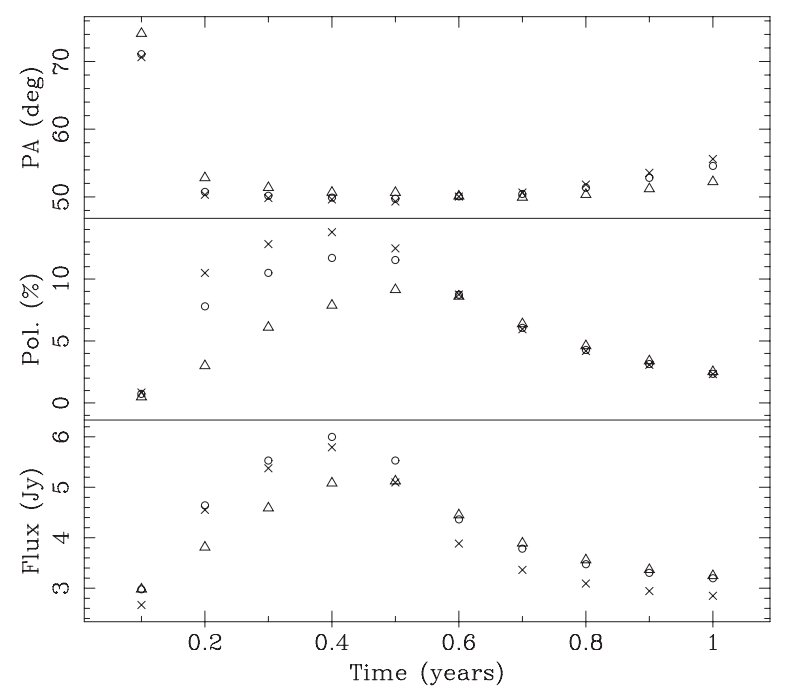

Figure 5. Oblique shock evolution using the parameter set "Run A" in Table 2. The panels and symbols correspond to those of Figure 1, showing from bottom to top: the total flux density, the fractional linear polarization, and the electric vector position angle, at three frequencies corresponding to the UMRAO observations at $14.5,8.0$, and $4.8 \mathrm{GHz}$ (crosses, circles, and triangles, respectively).

Assuming a forward shock $(\mathbf{F})$, implies that the shock plane moves over the underlying flow at Lorentz factor 6.7, which when viewed at an angle to the flow axis of $10^{\circ}$ would produce an apparent superluminal motion of the leading edge of $\beta_{\text {app }} \sim 6.5$. This is a significantly faster shock speed than adopted in modeling the 1980s outbursts, but the latter adopted a reverse $(\mathbf{R})$ shock model with the observer at a larger angle to the flow sense, guided by the low apparent speeds reported in the literature at the time. The current choice of parameters is guided by the much higher apparent speeds reported in the more recent literature (Lister et al. 2009), bearing in mind that over decades of activity the jet flow direction can change, either forward or reverse shocks might be evident at different epochs, and that shock events within a single source could exhibit a range of obliquities. As shown in Section 5.3, it is possible for oblique shocks to give rise to even higher percentage polarization, and thus more prominent, events than a transverse shock with similar parameters, for flows seen within tens of degrees of the line of sight.

Of the other parameters, optically thin spectral index is reasonably well constrained by observation and has been fixed accordingly. Others, such as flow opening angle and fiducial Lorentz factor, were picked on the basis of typical values discussed in the literature over many decades, to see if choice of such "plausible" values admits model light curves with the same characteristics as those observed. Cutoff Lorentz factors, shock width and optical depth (see Section 4), are adjusted in an attempt to reproduce light curves looking most like the example UMRAO data. The "order fraction" and orientations are subject to study in later sections.

Figure 5 shows the evolution of flux density, percentage polarization, and EVPA for this model, without the inclusion of retarded time effects. As described in Section 4 the calculations are done using dimensionless quantities; to "guide the eye," time and flux density have been arbitrarily scaled to values representative of those seen in the UMRAO data. The typical characteristics of UMRAO bursts described in Section 2.2- the fractional flux density increase $\Delta S /\langle S\rangle$, spectral evolution through a partially optically thick phase, percentage polarization with opacity/Faraday effects evident at the lowest frequency, and swing in EVPA by tens of degrees-are all reproduced. 
Table 2

Model Parameters

\begin{tabular}{lrrrr}
\hline \hline Parameter & Run A & \multicolumn{1}{c}{ Runs B } & Runs C & Runs D \\
\hline Semi-angle $(\mu)$ & 2.4 & & & \\
Spectral index $(\alpha)$ & 0.25 & & & \\
Fiducial LF $\left(\gamma_{c}\right)$ & 1000.0 & & & \\
Cutoff LF $\left(\gamma_{i}\right)$ & 50.0 & & & \\
Order multiple $(f)$ & - & & & \\
Bulk LF $\left(\gamma_{f}\right)$ & 2.5 & & & \\
Sense & F & & & \\
Shock width $(w)$ & 0.4 & & $45,90^{\circ}$ & \\
Compression $(\kappa)$ & 0.7 & & $20,40,60^{\circ}$ & \\
Obliquity $(\eta)$ & $45^{\circ}$ & & $30,50,70^{\circ}$ & \\
Orientation $(\psi)$ & $0^{\circ}$ & & & \\
Observer $\theta_{\text {obs }}$ & $10^{\circ}$ & & & \\
Observer $\phi_{\text {obs }}$ & $90^{\circ}$ & $0^{\circ}, 45^{\circ}, 90^{\circ}, 135^{\circ}$ & \\
& & $180^{\circ}, 225^{\circ}, 270^{\circ}, 315^{\circ}$ & & \\
& & & & \\
\end{tabular}

Note that in earlier modeling it was found that to fit the spectral characteristics of the polarized flux density, a fairly low cutoff thermal Lorentz factor $(\sim 20)$ was needed for some sources. Here the general characteristics of UMRAO outbursts are well reproduced with a value that means opacity effects dominate, with Faraday effects being only marginal: this implies that in general few low energy electrons are present in these sources.

While use of retarded time in the modeling would be necessary for detailed fits to data, the general characteristics of the total and polarized flux density light curves and spectral behavior, even without using retarded time, reproduce the behavior exhibited by the data (Section 2.2). Table 1(B) presents the model values corresponding to those derived from the UMRAO database and discussed in Section 2.2. A comparison of these is made in Section 6.

\subsection{Orientation}

Figure 6 shows models B1, B2, etc.- the same model as in Figure 5 (see Table 2), but for a range of observer orientation with respect to the shock plane. Recall that $\psi=0^{\circ}$, so that the shock normal lies in the $x-z$ plane of the Cartesian coordinate system. The observer orientations explored here $\left(0^{\circ}, 45^{\circ}, 90^{\circ}\right.$, $135^{\circ}, 180^{\circ}, 225^{\circ}, 270^{\circ}$, and $315^{\circ}$ ) correspond to starting with a view parallel to the $x$-axis, and then rotating around the jet so that by panel (e) the observer is "behind" the shock. As one would expect, the total flux density light curves are minimally changed by a change in azimuthal orientation. For an observer orientation within tens of degrees of $\phi_{\mathrm{obs}}=0^{\circ}$, orientation and aberration conspire to provide a more nearly "face-on" view of the shocked flow, and the percentage polarization is small. Indeed, at $\phi_{\text {obs }}=0^{\circ}$ the polarized flux density from the shock region cancels the small orthogonally polarized flux density associated with the axial field, as evidenced by the varied behavior in EVPA in panel (a). However, the percentage polarization is approaching $5 \%$ by orientations of $45^{\circ}$ and $335^{\circ}$, and exceeds $10 \%$ at peak over a large range of angles. The important conclusion is that azimuthal orientation does not play a major role in the total and polarized flux density outburst light curves; special conditions do not need to be invoked for polarizations of this order to be seen, and (subject to flow speed and polar orientation) most oblique structures will give rise to significant levels of polarized flux density. As the azimuthal angle does not play a significant role, a value of $90^{\circ}$ is adopted in what follows.

\subsection{Oblique versus Transverse Shocks}

In this section the run of percentage polarization with jet inclination for the transverse case is established, as a measure by which to judge the behavior in the oblique case; it is then shown that adopting an oblique shock does not radically change the behavior of percentage polarization-in fact leading to slightly higher values at some angles. This provides further evidence that the "shock in jet" model survives the introduction of oblique structures necessary to explain the temporal EVPA behavior seen in the UMRAO data and the evolution of features found in time sequences of VLBI maps.

Figure 7 shows models C1, C2, etc.- the same model as in Figure 5 (see Table 2), but contrasting transverse and oblique shocks for a range of observer orientation with respect to the flow axis. As orientation is changed, the free parameter $\tau$ is adjusted to ensure a similar spectral behavior of the total flux density. In panels (a)-(c) the shock is transverse, so the azimuthal location of the observer plays no role in determining appearance, and the observer is viewing at angles $20^{\circ}, 40^{\circ}$, and $60^{\circ}$, respectively, to the jet axis. Note that with increasing angle the decline in percentage polarization is rather slow. From Hughes et al. (1985), a flow with compression $\kappa=0.7$, seen edge-on, and in the absence of opacity and Faraday effects, would be expected to exhibit polarized emission $\sim 25 \%$, dropping to $\sim 8 \%$ at an angle of $50^{\circ}$. Given the modest Lorentz factor of the shocked flow in the observer frame, $\sim 3$, radiation from this angle outside the critical cone of the flow (namely, at $140^{\circ}$ to the flow axis in the flow frame) would be seen by the observer viewing at $50^{\circ}$ to the flow axis, an orientation spanned by panels (b) and (c) in the figure. In the simple transverse case, for this level of compression, quite high levels of polarization will be seen well beyond those values of viewing angle usually adopted in blazar modeling. Table 1(C) presents the model values for Run C1, corresponding to those derived from the UMRAO database and discussed in Section 2.2. The only large difference compared with the values presented in Table $1(\mathrm{~B})$ is in the jump in EVPA as expected, as well as in the spread in EVPA at outburst end.

In panels (d)-(f) the original oblique shock is viewed at angles $30^{\circ}, 50^{\circ}$, and $70^{\circ}$ to the jet axis, for an azimuthal orientation of $90^{\circ}$. (For an azimuthal orientation of $0^{\circ}$ the situation will be approximately-subject to flow deflection and different aberration-as for the transverse case, modulo an angular offset.) It can be seen that a high level of polarized emission persists to quite large angle from the flow axis, a result 

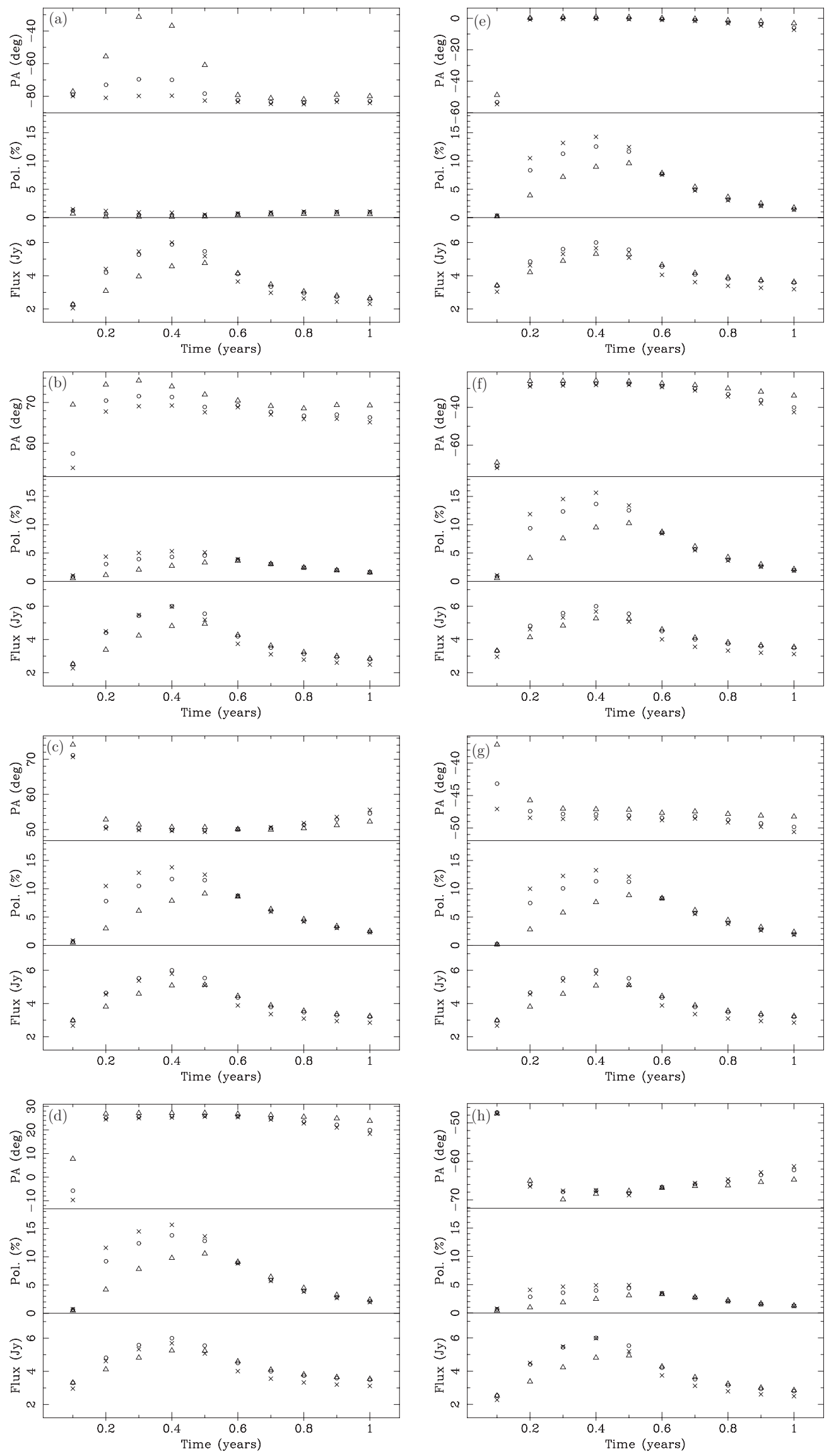

Figure 6. Same model as shown in Figure 5, for a range of observer orientations with respect to the shock plane. From panel (a) the azimuthal angle of the observer is $0^{\circ}, 45^{\circ}, 90^{\circ}, 135^{\circ}, 180^{\circ}, 225^{\circ}, 270^{\circ}$, and $315^{\circ}$. (Note that in these models the zero point for the EVPA is arbitrary and can change from plot to plot-only the range in values is significant.) 

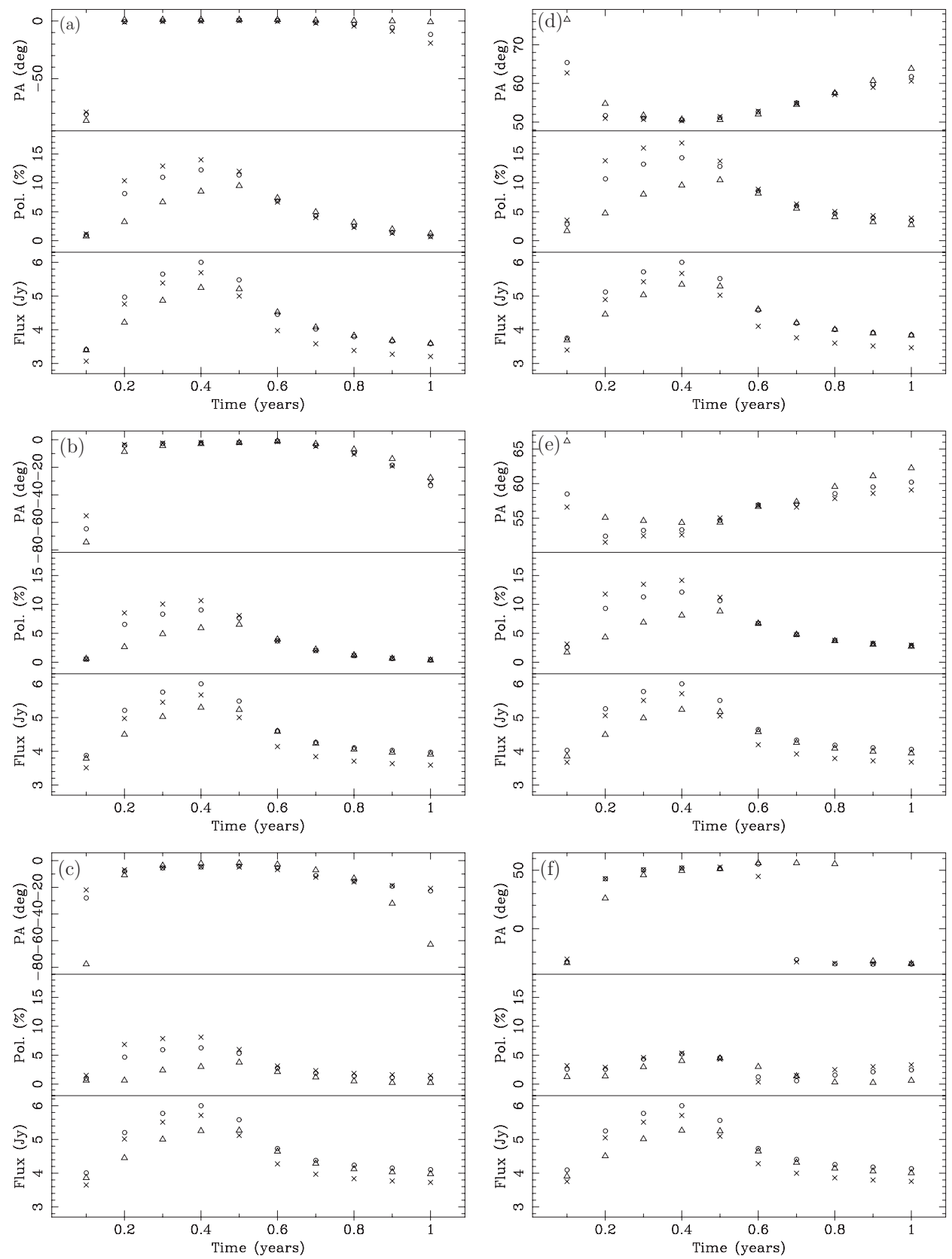

Figure 7. Comparison of a transverse shock model with that of oblique Run A, as shown in Figure 5, for a range of polar angles. In the left panels the shock orientation has been adjusted to be transverse to the flow, and in panels (a)-(c) the observer is viewing at angles $20^{\circ}, 40^{\circ}$, and $60^{\circ}$ to the jet axis. The right panels are for the oblique case, and in panels (d)-(f) the observer is viewing at angles $30^{\circ}, 50^{\circ}$, and $70^{\circ}$ to the jet axis. (The range of angles is chosen to optimally display variation with observer orientation. Note that in these models the zero point for the EVPA is arbitrary and can change from plot to plot—only the range in values is significant.)

related to the geometrical effect that as the shock becomes more nearly parallel to the flow, rotation in polar angle has no effect on the appearance of a flow seen initially nearly edge-on. As with azimuthal orientation, it is concluded that special conditions do not need to be invoked, and that the observed levels of percentage polarization can be seen for oblique shocks for a significant range of observer orientation with respect to the flow axis.

\subsection{Magnetic Field}

Figure 8 shows models D1, D2, etc.- the same model as in Figure 5 (see Table 2), but for a range of "order multiple," a measure of the importance of an ordered component of magnetic field. The values explored here $(0.1,0.3,1.0$, and
3.0) correspond to moving from an essentially random field to an almost totally ordered field. A fixed multiple at each point within the flow is assumed; as discussed in Section 3.2.2, trials show that the details of how the relative strength of these two components is modeled do not have a significant influence on the results.

As noted by Lyutikov et al. (2005), low levels of percentage polarization may result even if the emitting volume contains wholly ordered magnetic field, and that is evident in the values seen here outside of shock events-significant compared with the random field case, but low relative to the maximum value for optically thin synchrotron radiation. Careful choice of field topology might reduce the value further, but as argued in Section 3.2.2, the adopted one appears to be the most physically plausible. 

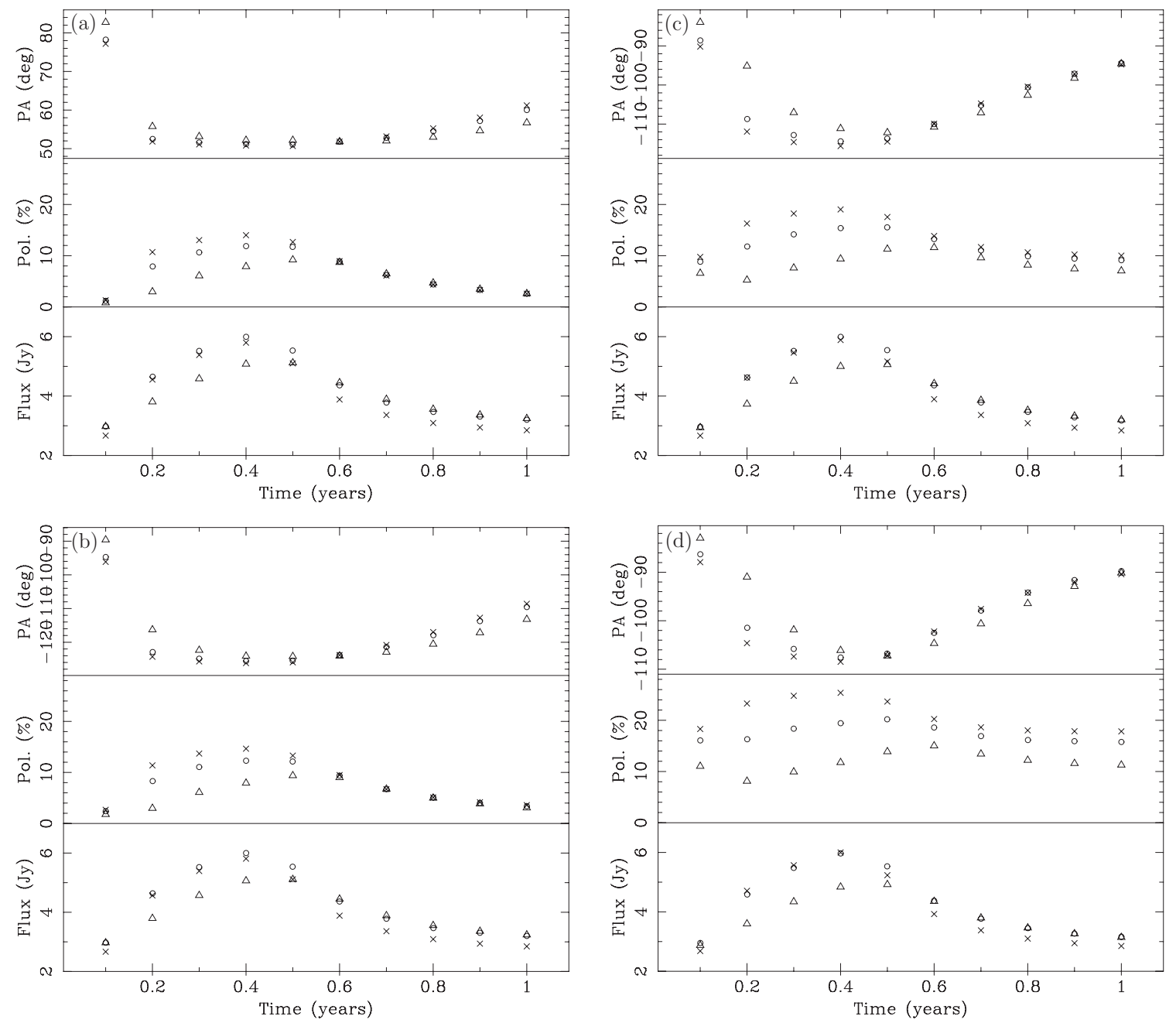

Figure 8. Same model as shown in Figure 5, for a range of ordered to random magnetic field strengths. From panel (a) the "order multiple" $f$ is $0.1,0.3,1.0$, and 3.0, so that in panel (a) the random field dominates, while in panel (d) the ordered field dominates. (Note that in these models the zero point for the EVPA is arbitrary and can change from plot to plot-only the range in values is significant.)

The nature of the outburst in percentage polarization differs markedly between the case of polarization induced by compression of a random field, as seen in panel (a) of Figure 8, and the case of polarization induced by asymmetric compression of an ordered field, as seen in panel (d). In the latter case the amplitude of the rise is limited (by less than a factor of $\sim 1.5$ ), with a strong frequency dependence unlike either that seen in panel (a) or observed outbursts; the frequency dependence arises because at the lowest frequency opacity causes polarized emission to be dominated by counter-directed magnetic field arcs near to the jet boundary. The behavior of the EVPA is also markedly different between the cases. For the random field example the EVPA swings abruptly, and similarly at all three frequencies, to an approximately constant value during the long decline in outburst. In the ordered field example there is a strongly frequency-dependent decline, followed by an immediate recovery, and no distinct plateau of EVPA during outburst. The evolution shown for the cases of a predominantly ordered (helical) field neither matches what has been seen in the evolution of the linearly polarized flux density during wellresolved, distinct events that have been followed in detail, nor is the spectral evolution of the position angle in the rise portion of the event generally consistent with the observations. Based on this evidence, it is concluded that the magnetic field within the emitting region cannot be largely of the ordered helical type.

\section{CONCLUSIONS}

The "shock in jet" model for centimeter-waveband blazar variability has been revisited, allowing for arbitrary shock orientation with respect to the jet flow direction, and both random and ordered magnetic field. Oblique shocks can explain events with swings in polarization position angle much less than the $90^{\circ}$ associated with transverse structures, while retaining the general characteristics of outbursts, including spectral behavior and level of peak percentage polarization.

Specifically, as can be seen from Table 1, the model reproduces the temporal behavior of the UMRAO data: the trend in spectral evolution through an opaque phase, while remaining quite flat throughout the burst evolution; significant levels of percentage polarization (but far less than the maximum permitted for a homogeneous source); the magnitude of temporal evolution seen in EVPA; and the frequency spread in EVPA during outburst of order degrees. The range of models provided by varying the observer orientation with respect to the shock plane (Section 5.2, Figure 6) displays the same general characteristics, but a range of percentage polarization from almost zero to $\sim 20 \%$, corresponding to the range seen in UMRAO data (Section 2.2).

For certain azimuthal orientations with respect to the shock normal the percentage polarization during outburst is low-not 
much more than that seen in the quiescent state-but in general, levels of polarization commensurate with that seen in UMRAO monitoring data occur, indicating that oblique shocks can explain the data without recourse to special observer orientation. Similarly, for the flow parameters adopted here (modest Lorentz factor, leading to modest aberration) oblique shocks can give rise to the observed levels of percentage polarization for a broad range of orientation with respect to the flow axis, as is the case for a transverse shock with similar parameters. As found by Hughes et al. (1989b), only modeling of the detailed outburst profile for specific events with the inclusion of retarded time effects (in addition to matching the precise level of polarization achieved at peak outburst) will better constrain the viewing angle.

Models dominated by a force-free, minimum energy magnetic field configuration (essentially helical) display a limited rise in percentage polarization, and a frequency-dependent swing in polarization position angle not in agreement with the results of single-dish monitoring programs, implying that the field cannot be dominated by an ordered component with the character of a force-free flux rope in the quiescent state. This is in agreement with the conclusions of a number of studies (discussed in Section 3.2) that argue against a predominantly helical field configuration within the emitting region.

Outbursts well explained by the "shock in jet" model are present during $\gamma$-ray flaring in several sources, supporting the idea that shock events are associated with activity from the radio to $\gamma$-ray bands. Some $\gamma$-ray flares might be associated not with propagating shocks, but rather with propagating particle density/magnetic field enhancements that encounter stationary structures such as a recollimation shock (Agudo et al. 2011). Such events are best explained if the magnetic field of the propagating enhancement is random, and so our conclusion that the quiescent flow of blazars is predominately random provides support for this explanation.

We thank the referee for a careful reading of the paper, and many detailed, thoughtful, and constructive comments. This work was made possible by support from NSF grant NSF0607523, NASA Fermi grants NNX09AU16G and NNX10AP16G, and by support for the operation of UMRAO from the University of Michigan. This research has made use of data from the MOJAVE database that is maintained by the MOJAVE team (Lister et al. 2009).

\section{REFERENCES}

Abdo, A. A., et al. 2010a, ApJ, 721, 1425

Abdo, A. A., et al. 2010b, ApJ, 722, 520

Agudo, I., et al. 2011, ApJ, 726, L13

Aller, M. F., Aller, H. D., \& Hughes, P. A. 2010, arXiv:1012.1359

Broderick, A. E., \& McKinney, J. C. 2010, ApJ, 725, 750
Cawthorne, T. V., \& Cobb, W. K. 1990, ApJ, 350, 536

Contopoulos, I., Christodoulou, D. M., Kazanas, D., \& Gabuzda, D. C. 2009, ApJ, 702, L148

Denn, G. R., Mutel, R. L., \& Marscher, A. P. 2000, ApJS, 129, 61

Eilek, J. A., \& Hughes, P. A. 1991, in Beams and Jets in Astrophysics, ed. P. A. Hughes (Cambridge: Cambridge Univ. Press), 428

Gabuzda, D. C., Murray, É., \& Cronin, P. 2004, MNRAS, 351, L89

Gabuzda, D. C., Murray, E., \& Cronin, P. 2005, Balt. Astron., 14, 363

Homan, D. C., Lister, M. L., Aller, H. D., Aller, M. F., \& Wardle, J. F. C. 2009, ApJ, 696, 328

Hughes, P. A. 2005, ApJ, 621, 635

Hughes, P. A., Aller, H. D., \& Aller, M. F. 1985, ApJ, 298, 301

Hughes, P. A., Aller, H. D., \& Aller, M. F. 1989a, ApJ, 341, 54

Hughes, P. A., Aller, H. D., \& Aller, M. F. 1989b, ApJ, 341, 68

Hughes, P. A., Aller, H. D., \& Aller, M. F. 1991, ApJ, 374, 57

Hughes, P. A., Aller, H. D., \& Aller, M. F. 1992, ApJ, 396, 469

Jones, T. W. 1988, ApJ, 332, 678

Jones, T. W., Rudnick, L., Aller, H. D., Aller, M. F., Hodge, P. E., \& Fiedler, R. L. 1985, ApJ, 290, 627

Komissarov, S. S., Barkov, M. V., Vlaha kis, N., \& Königl, A. 2007, MNRAS, 380,51

Königl, A., \& Choudhuri, A. R. 1985, ApJ, 289, 173

Kovalev, Y. Y., et al. 2005, AJ, 130, 2473

Kovalev, Y. Y., et al. 2009, ApJ, 696, L17

Laing, R. A. 1996, in ASP Conf. Ser. 100, Energy Transportation in Galaxies and Quasars, ed. P. E. Hardee, A. H. Bridle, \& J. A. Zensus (San Francisco, CA: ASP), 241

Laing, R. A., Parma, P., de Ruiter, H. R., \& Fanti, R. 1999, MNRAS, 306, 513

Leahy, J. P. 1991, in Beams and Jets in Astrophysics, ed. P. A. Hughes (Cambridge: Cambridge Univ. Press), 100

Lind, K. R., \& Blandford, R. D. 1985, ApJ, 295, 358

Lister, M. L., et al. 2009, AJ, 138, 1874

Lundquist, S. 1950, Ark. Fys., 2, 361

Lyutikov, M., Pariev, V. I., \& Gabuzda, D. C. 2005, MNRAS, 360, 869

Mahmud, M., \& Gabuzda, D. 2008, in ASP Conf. Ser. 386, Extragalactic Jets: Theory and Observation from Radio to Gamma Ray, ed. T. A. Rector \& D. S. De Young (San Francisco, CA: ASP), 494

Mahmud, M., Gabuzda, D. C., \& Bezrukovs, V. 2009, MNRAS, 400, 2

Marscher, A. P. 2006, in AIP Conf. Proc. 856, Relativistic Jets: The Common Physics of AGN, Microquasars, and Gamma-Ray Bursts, ed. P. A. Hughes \& J. N. Bregman (Melville, NY: AIP), 1

Marscher, A. P., \& Gear, W. K. 1985, ApJ, 298, 114

Marscher, A. P., \& Jorstad, S. G. 2011, ApJ, 729, 26

Marscher, A. P., et al. 2010, ApJ, 710, L126

McKinney, J. C. 2006, MNRAS, 368, 1561

McKinney, J. C., \& Narayan, R. 2007, MNRAS, 375, 513

O'Sullivan, S. P., \& Gabuzda, D. C. 2008, in ASP Conf. Ser. 386, Extragalactic Jets: Theory and Observation from Radio to Gamma Ray, ed. T. A. Rector \& D. S. De Young (San Francisco, CA: ASP), 284

Richards, J. L., et al. 2010, ApJS, submitted (arXiv:1011.3111)

Rogachevskii, I., \& Kleeorin, N. 2003, Phys. Rev. E, 68, 036301

Rusk, R. E. 1988, Ph D thesis, Univ. Toronto

Smith, M. D. 1984, MNRAS, 211, 767

Taylor, G. B., \& Zavala, R. 2010, ApJ, 722, L183

Tribble, P. C. 1991, MNRAS, 253, 147

Valtaoja, E., Lähteenmäki, A., Teräsranta, H., \& Lainela, M. 1999, ApJS, 120, 95

Valtaoja, E., \& Teräsranta, H. 1995, A\&A, 297, L13

Valtaoja, E., \& Teräsranta, H. 1996, A\&AS, 120, 491

Yousef, T. A., Heinemann, T., Schekochihin, A. A., Kleeorin, N., Rogachevskii, I., Iskakov, A. B., Cowley, S. C., \& McWilliams, J. C. 2008, Phys. Rev. Lett., 100,184501 